\title{
Orphée, figure du merveilleux dans l'opéra baroque
}

\author{
Raphaëlle Legrand (Université de la Sorbonne - Paris IV, Paris, França) \\ raphaelle.legrand@paris-sorbonne.fr
}

Résumé: Le personnage d'Orphée est présent dans des œuvres lyriques majeures du début et à la fin de la période baroque (Peri, Caccini, Monteverdi, Gluck). II apparaît également dans des opéras (Rossi, Charpentier, L. de Lully), ballets (J.B. Lully) ou cantates (Rameau) où les diverses modalités du merveilleux musical baroque s'expriment à travers ce mythe polymorphe. Orphée peut aussi être vu comme une métaphore de la synthèse des arts propre à l'opéra.

Mots-clés: cantate; merveilleux; musique baroque; Orphée, opéra.

\section{Orfeu, figura do maravilhoso na ópera barroca}

Resumo: 0 personagem de Orfeu está presente nas maiores obras líricas do início ao final do período barroco (Peri, Caccini, Monteverdi, Gluck). Ele aparece também nas óperas (Rossi, Charpentier, L. de Lully), balés (J.B. Lully) ou cantatas (Rameau) nas quais as diversas modalidades do maravilhoso musical barroco se exprime através deste mito polimorfo. Orfeu pode também ser visto como uma metáfora da síntese das artes, própria da ópera.

Palavras-chave: cantata; maravilhoso; música barroca; Orfeu, ópera.

\section{1 - Introduction}

Parmi tous les héros, toutes les héroïnes mythiques ou historiques convoqués sur la scène des théâtres lyriques, Orphée est sans doute le personnage le plus emblématique du merveilleux musical, son chant magique lui permettant de transgresser la loi des mortels, de passer d'un monde à un autre, d'attendrir jusqu'aux divinités infernales ${ }^{1}$. Et ce n'est sans doute pas un hasard si le mythe d'Orphée est présent à la fois au moment de la naissance de l'opéra, en Italie au début du xvile siècle, et lorsque Gluck opère une mutation radicale entre l'esthétique des passions et celle du sentiment, au milieu du xvIIIe siècle. Des Euridice florentines et de L'Orfeo de Monteverdi à Orfeo ed Euridice de Gluck, le personnage d'Orphée est un bon guide pour contempler et comprendre les merveilles du baroque musical.

\section{2 - Puissance d'un mythe polymorphe}

La légende d'Orphée est connue par les poètes et les musiciens des XVIle et XVIIle siècles à travers deux grands textes : les Métamorphoses d'Ovide et les Georgiques de Virgile. On trouve dans les deux versions les mêmes éléments fondamentaux : les noces d'Orphée et Eurydice interrompues par la mort de la jeune femme, mordue par un serpent; la descente d'Orphée au Enfers, qui fléchit Proserpine et Pluton par la puissance de son chant; la condition posée pour ramener Eurydice au monde des vivants (ne pas la regarder en chemin); le difficile retour, l'impatience d'Orphée qui se retourne et perd de nouveau sa bien-aimée; le désespoir du musicien qui renonce alors au commerce des femmes; sa mort, déchiré par les Bacchantes qui se vengent de son indifférence. Mais Virgile introduit un élément nouveau : selon lui, c'est en fuyant le berger Aristée qu'Eurydice a été mordue par le serpent. Par ailleurs, Ovide conclut, contrairement à Virgile, sur la réunion ultime des amants dans le royaume des morts. Et sur bien des points, les deux récits divergent légèrement.

Telle qu'elle apparaît dans les sources latines, la légende d'Orphée présente des zones d'ombre et de lumière et le personnage n'est pas sans contradictions. Orphée peut être vu comme un héros courageux qui brave les pires dangers, mais aussi comme un homme qui manque de maîtrise sur ses passions et qui perd par trop d'impatience tout le bénéfice de son héroïsme. Orphée peut être vu comme le modèle de l'époux fidèle, risquant sa vie pour sauver la femme qu'il aime, renonçant à tout autre 
amour; il est présenté aussi par Ovide comme l'inventeur de l'homosexualité masculine. Il est celui dont la voix magique émeut les dieux, les hommes et les ombres, charme les animaux, les plantes et les rochers, brouillant les frontières entre l'animé et l'inanimé, entre la vie et la mort. II est montré enfin comme un propagateur des mystères de Bacchus, tout en étant protégé par Apollon.

A l'époque baroque, il s'agissait donc de choisir dans ce mythe polymorphe ce qui était convenable, ce qui pouvait coïncider avec une morale chrétienne et un dénouement satisfaisant. Transformer le mythe en une structure dramatique cohérente impliquait des choix et des distorsions. Ainsi, l'épisode des Bacchantes fut rarement mis en scène. On préféra parfois renoncer à la deuxième mort d'Eurydice (lavant ainsi Orphée de toute faute) ou, plus souvent, faire intervenir un dieu, Apollon, Jupiter ou l'Amour, pour sauver le malheureux amant tenté par le suicide et lui proposer une sublimation de sa douleur, voire l'élever au rang de constellation. Mais ces corrections apportées aux récits d'Ovide et de Virgile n'empêchaient pas les spectateurs, qui connaissaient parfaitement les sources antiques, de superposer dans leur esprit les différentes versions, dans un jeu sur les doubles sens auquel ils étaient habitués et qui nous échappe souvent.

Quoi qu'il en soit, les aventures d'Orphée, avec l'opposition du monde pastoral des vivants et du monde souterrain des morts, la profusion de personnages divins ou fabuleux, et la magie de la voix du chantre de la Thrace, cumulaient suffisamment d'éléments merveilleux pour former de magnifiques spectacles.

\section{3 - Déclinaisons musicales du mythe}

Le personnage d'Orphée est donc présent au moment même où s'invente l'opéra, à Florence et à Mantoue. Les premiers opéras conservés, en effet, content I'histoire du célèbre chanteur : les deux Euridice de Jacopo Peri et Giulio Caccini en 1600 à Florence, composées toutes deux sur le même poème d'Ottavio Rinuccini, l'Orfeo de Monteverdi en 1607 à Mantoue, sur un poème d'Alessandro Striggio. Si l'on conserve la trace de précédents spectacles, comme la Dafne de Rinuccini et Peri en 1598, fondée sur un autre épisode des Métamorphoses d'Ovide et dont la musique est perdue, le destin d'Orphée et d'Eurydice reste le sujet principal des premiers opéras, fruits des recherches, des débats et des rêves érudits d'humanistes qui pensaient ressusciter la tragédie antique, mais qui en réalité inventaient un nouveau continent esthétique: le baroque musical.

Baroques, ces opéras l'étaient principalement par l'emploi du récitatif, déclamation musicalisée accompagnée par la basse continue. II faut mesurer la force innovatrice de ce chant soliste dans le contexte de la tradition polyphonique, où les sentiments d'un seul étaient exprimés par quatre ou cinq parties chantées. Le chanteur-acteur se présente alors comme un individu isolé, et par la force de l'illusion théâtrale, les passions qu'il assume semblent être les siennes propres, tout comme celles du public qui compatit à ses malheurs. On comprend que le personnage d'Orphée, chanteur fabuleux se présentant lui aussi seul, armé de sa lyre, devant les portes des Enfers pour passer dans l'autre monde grâce à la puissance de son art, ait été la figure emblématique de cette mutation musicale.

Les Euridice florentines et l'Orfeo de Monteverdi sont par ailleurs des spectacles de cour, présentés devant un public restreint et cultivé. Ces œuvres sont imprégnées de philosophie néo-platonicienne et la musique y joue un rôle éminent : celui d'une harmonie aussi terrestre que divine, qu'Orphée symbolise aussi. Le prologue des Euridice de Rinuccini présente le personnage de la Tragédie, mais celui de Striggio et de Monteverdi met en avant l'allégorie de la Musique, qui gouverne les âmes et les cieux. En cela également ces opéras sont typiquement baroques, par leur souci d'incarner une pensée philosophique, de la mettre en scène, de la transformer en images, en gestes et en sons, pour mieux persuader l'auditoire.

L'Euridice de Rinuccini, mise en musique par Peri et par Caccini, se coule dans une simple structure en trois actes. Le premier acte présente dans un cadre pastoral la joie des noces d'Orphée, troublée soudain par le récit de la mort d'Eurydice; le deuxième montre Orphée fléchissant les dieux infernaux; le troisième retrouve le cadre initial pour un retour triomphal des deux amants. Dans sa simplicité, l'intrigue favorise les effusions poétiques des personnages et du chœur, très présent, dans un style plus lyrique, encore, que dramatique.

L'Orfeo de Striggio et Monteverdi est plus ample que les opéras florentins. L'œuvre se développe sur cinq actes précédés d'un prologue et organisés suivant une structure symétrique, un goût pour l'architecture poétique et sonore que l'on retrouve jusque dans le détail des formes musicales. Le premier acte montre la joie des noces d'Orphée et d'Euridice dans un contexte pastoral. Le deuxième est consacré à la mort de la jeune femme, sous forme de récit, et la détermination d'Orphée d'aller chercher son épouse au royaume des morts. Le troisième est le centre de l'opéra, la confrontation entre Orphée et Caron, et le déploiement de virtuosité et d'expressivité du chanteur dans son invocation "Possente spirto". Le quatrième acte est symétrique au deuxième : Orphée désobéit, se retourne et perd de nouveau Euridice. Le dernier acte est celui d'une sublimation : à une première version où le musicien était tué par les Bacchantes, et dont la musique est perdue, se substitue une seconde, où Apollon intervient pour arrêter le suicide de l'amant éploré et l'inviter à dépasser sa douleur, et même son amour terrestre, pour s'élever avec lui jusqu'au monde spirituel et parvenir au véritable et pur amour.

Après ces trois opéras initiaux, le genre va se propager rapidement dans toute I'Italie. On bâtit des théâtres qui lui sont spécialement consacrés. A Venise, l'opéra s'ouvre à un plus large public; à Rome, il est mis en scène avec 
faste par des dignitaires ecclésiastiques qui ne dédaignent pas d'écrire des livrets. Le comique vient alors se mêler aux références mythologiques, les personnages secondaires se multiplient, les intrigues sont d'une extrême fantaisie et jouent avec une grande désinvolture avec les épisodes bien connus du public. Le merveilleux est à la fois convoqué et mis à distance, avec une rare subtilité. Les passions les plus contrastées se succèdent rapidement, on rit, on pleure, et on s'étonne continuellement.

Dans cette veine, il faut citer l'Orfeo de Luigi Rossi, sur un livret de Francesco Buti, un opéra dans la pure tradition romaine, créé pourtant à Paris en 1647 à la demande du ministre Mazarin. La simple histoire d'Orphée y est enrichie de nombreux épisodes. Les deux premiers actes, dans un opéra qui en compte trois, sont ainsi consacrés aux entreprises d'Aristée pour empêcher les noces d'Eurydice avec Orphée. On voit par exemple Vénus se costumer en vieille femme pour prêcher l'inconstance à la jeune fiancée. En revanche, la plainte d'Orphée est une page véritablement poignante. La multiplication des personnages, dieux, allégories et comparses comiques crée un effet de profusion qui est aussi un des traits du baroque.

En France, le personnage d'Orphée apparaît épisodiquement dans un ballet de cour où danse Louis XIV, le Ballet des Muses: Orphée chante une "plainte " qui montre la capacité du Florentin Jean-Baptiste Lully, futur créateur de l'opéra français, à écrire dans le style pathétique italien.

Lully cependant ne choisira pas le sujet d'Orphée pour ses tragédies en musique, préférant en général des héros plus guerriers, offrant ainsi au roi-soleil l'image héroïque et galante qu'il cherche à promouvoir. Ce n'est peut-être pas un hasard si le sujet apparaît, dans l'opéra public français, à un moment de crise : peu après la mort de Lully, alors que Louis XIV s'est désintéressé des spectacles et que le genre lyrique doit trouver d'autres raisons d'être. C'est en 1690 que Louis de Lully, l'un des jeunes fils du célèbre Surintendant de Louis XIV, compose un Orphée (sur un livret de Michel du Boullay) qui ne rencontre pas un grand succès mais qui est très représentatif des opéras de cette époque, avec son dénouement tragique, Orphée étant tué par des Bacchantes.

Quelques années auparavant, vers 1686-1687, Marc-Antoine Charpentier a traité le sujet, d'abord dans une brève cantate (Orphée descendant aux Enfers, H 471), puis dans un petit opéra, La descente d'Orphée aux Enfers (H 488), tous deux composés sur des poèmes restés anonymes. $L a$ descente d'Orphée aux Enfers est prévue pour des représentations privées chez madame de Guise. Si elle reprend les traits spécifiques de l'opéra français de Lully, notamment la présence de ballets, elle en use avec souplesse et liberté : l'instrumentation est légère mais variée (notons l'emploi des violes pour figurer la lyre d'Orphée) et Charpentier opère avec bonheur la fusion des styles français et italien. Loin du grand appareil de l'opéra public parisien, l'Académie royale de musique, cet opéra de petites proportions manifeste la vitalité de la création musicale en dehors des grands centres officiels. Les deux actes de La descente d'Orphée aux Enfers représentent la mort d'Eurydice, fauchée en plein bonheur, puis le voyage d'Orphée aux Enfers et sa magnifique prière à Pluton. L'œuvre se clôt avant le voyage de retour, qu'Orphée aborde avec crainte, la condition qui lui est faite étant bien dure pour un amant. On imagine aisément qu'un troisième acte ait pu être conçu, mais actuellement perdu, complétant l'histoire.

C'est un autre merveilleux baroque qui est convoqué dans cette délicate miniature : moins imposant que les œuvres dont nous avons parlé, s'orientant vers le style plus allégé et plus intime qui prévaudra au XVIIle siècle, mais non moins émouvant. Ainsi, aux Enfers, le chant d'Orphée ne fait pas qu'attendrir les divinités locales, il allège momentanément les peines des damnés, comme Tantale ou Ixion, qui lui diront adieu avec nostalgie au moment de son retour dans le monde des vivants. Enfin le plaidoyer d'Orphée devant le dieu des Enfers est construit suivant une savante rhétorique, partant du silence de la voix qui manque à l'amant angoissé, pour parvenir peu à peu à une argumentation triomphante se prévalant de l'intercession de Proserpine pour fléchir le sombre Pluton.

Ce style préfigure le succès du mythe d'Orphée dans la cantate française des premières décennies du XVIIle siècle, ces œuvres pour une voix soliste et quelques instruments de Nicolas Bernier, Louis-Nicolas Clérambault, Jean-Philippe Rameau et d'autres moins connus. Dans ces courtes pièces de chambre non représentées, c'est le récitatif qui assume la narration, plante le décor, rappelle la situation à un public qui en connaît parfaitement les sources. L'action se cristallise en trois ou quatre airs où les personnages s'expriment au style direct. Datant de 1721, la cantate Orphée de Rameau joue sur les contrastes les plus absolus. L'épisode retenu par le poète anonyme est celui qui justement manque au petit opéra de Charpentier : Orphée quitte les Enfers suivi d'Eurydice, encouragé par un " air gai " montrant une nuée d'Amours qui chantent sa victoire. Mais on sombre rapidement dans le drame : dans un puissant récitatif accompagné, Orphée se trouble, se retourne, voit mourir Eurydice. Son invocation à l'Amour n'y fait rien, il n'a plus qu'à mourir luimême. Mais alors que nous sommes profondément émus par la puissance évocatrice de I'harmonie ramiste, l'air final propose avec détachement une morale libertine, qui joue avec équivoque sur la maîtrise du temps qui caractérise les parfaits amants. Encore une fois, le merveilleux (ici évoqué plus que représenté) est à la fois nécessaire et mis à distance. C'est le propre de l'esthétique baroque de créer l'illusion puis de soulever le voile sur la machinerie qui l'a rendue possible, de mouvoir les passions et d'obliger le spectateur ou l'auditeur à analyser en lui-même le phénomène. II y a là une jouissance intellectuelle bien particulière, un enseignement qui prend le masque du divertissement et recouvre avec élégance sa portée didactique par des ornements en apparence de 
pure fantaisie. Plaire, émouvoir et instruire, les buts affichés de la rhétorique sont constamment à l'œuvre dans les spectacles en musique baroques, dans l'opéra le plus ostentatoire comme dans le petit théâtre imaginaire que représente la cantate.

C'est cette mise à distance ludique et profonde qui va être remise en cause radicalement par la réforme de Christoph Willibald Gluck et de son librettiste Ranieri Calzabigi. Paradoxalement, Gluck et Calzabigi semblent revenir à certains traits d'une tradition baroque ancienne, dans leur azione teatrale créée à la cour de Vienne en 1762, Orfeo ed Euridice. Pour régénérer l'opera seria triomphant à son époque, qui a remplacé les sujets mythologiques par des épisodes historiques et qui a évincé le chœur et le ballet au profit des chanteurs solistes, Gluck paraît se référer aux premiers opéras du XVIle siècle et à ce que l'opéra français de son temps pouvait receler d'archaïsmes. Mais ce retour à l'origine de l'opéra et à ses mythes n'est pas une restauration : Gluck (et les autres réformateurs comme Jommelli et Traetta) ne se situe plus dans la profusion, l'ornemental, la rhétorique et la mise à distance. Le mythe est pour lui avant tout simplicité et nudité. II étire les épisodes où le chœur et le ballet entourent le soliste pour créer de longues plages temporelles, statiques mais d'un effet scénique très puissant, suivant l'esthétique du tableau qui commence à prévaloir au théâtre. Ainsi la confrontation entre Orphée et les spectres qui gardent les portes des Enfers, opposant les mélodies suaves et attendrissantes du chanteur aux les refus brutaux du chœur, cet épisode remplit la moitié du second acte. Non content de résumer l'intrigue, Gluck épure les lignes mélodiques des solistes, dans un souci très néo-classique, tout en traitant les masses chorales et orchestrales avec simplicité et efficacité. II ne cherche pas à susciter l'enchantement du merveilleux baroque mais l'empathie avec un personnage dont on représente le théâtre intérieur : l'Amour qui vient conseiller Orphée, unique messager d'un Olympe bien lointain, est-il un dieu ou seulement une allégorie ? Les gardiens des portes des Enfers sont-ils des spectres ou la métaphore des mouvements obscurs de l'âme ? Le théâtre des passions devient celui du sentiment, voie ouverte vers l'évolution de l'opéra au XIXe recherchant toujours plus de réalisme et s'orientant vers l'analyse psychologique.

\section{4 - Orphée, métaphore du merveilleux lyrique} S'il fallait synthétiser les usages du merveilleux dans ces Orphée divers, nous pourrions déjà nous intéresser aux espaces scéniques ouverts par le mythe. Les opéras baroques convoquant les dieux superposent souvent trois espaces : les mortels évoluent sur la scène et entrent par les coulisses dans un mouvement horizontal, les dieux occupent l'espace au-dessus de la scène, puisqu'ils descendent des cintres et y retournent sur leurs machines volantes dans un mouvement vertical ou parfois oblique, les divinités infernales occupent un sous-sol imaginaire et surgissent des dessous du théâtre par des trappes. Tout l'espace, scénique et hors-scène, est saturé de personnages, mais suivant une minutieuse hiérarchie.
L'histoire d'Orphée présente l'avantage de justifier un acte entier dans les Enfers. Le clivage des espaces (monde humain et monde souterrain) est alors projeté dans le temps, dans la succession des actes, dans le voyage d'Orphée d'un monde à un autre. Dans l'Orfeo de Monteverdi et celui de Rossi, le dénouement convoque également l'espace des dieux, soit qu'Apollon élève à son niveau Orphée, dans tous les sens du terme, soit que le chanteur et sa lyre soient transformés en constellation.

On pourrait opposer enfin le monde naturel (celui des mortels) au monde surnaturel (celui des morts) mais en réalité le climat pastoral où évoluent les humains n'est pas non plus exempt de merveilleux. L'insertion par Virgile de l'épisode d'Orphée dans ses Georgiques incite les auteurs baroques à brosser longuement le tableau du bonheur d'Orphée et d'Eurydice dans un cadre champêtre. Mais nul réalisme : les nymphes, et parfois même les satyres se mêlent aux bergers. Car la pastorale à cette époque représente une sorte d'âge d'or, une Arcadie perdue où l'amour est durable et sans nuages. Ce climat pastoral doit donc être compris comme une métaphore de l'amour heureux et sincère d'Orphée et Eurydice, brisé par la mort. La question sera surtout de savoir si le dénouement ramène les amants dans le lieu idyllique de leurs amours (Gluck), ou si l'épreuve du voyage aux Enfers oriente Orphée vers un autre destin, tragique (Louis de Lully) ou glorieux (Monteverdi).

Une autre approche du merveilleux, plus spécifiquement musicale, serait d'interroger le chant d'Orphée. Vecteur de l'art d'Orphée, sa voix elle-même est merveilleuse, elle réalise des prodiges. La difficulté, pour les compositeurs, est de la faire entendre comme chant, au sein d'un spectacle entièrement chanté. II est possible de lui écrire un air tout à fait exceptionnel, dans ses dimensions, son style, son ornementation, comme le "Possente spirto " de Monteverdi, qui tranche avec ce qui précède et qui suit. II est utile également de faire appel à un interprète renommé, comme le célèbre castrat Atto Melani pour l'Orfeo de Rossi. Car l'Orphée légendaire est à la fois poète, musicien, chanteur. Comme les poètes antiques, il improvise vers et musique en s'accompagnant lui-même sur la lyre. Sur la scène d'un opéra baroque, cette fonction unifiée se fragmente : elle est assumée à la fois par le poète qui conçoit le livret, par le compositeur qui invente la musique, par l'interprète qui incarne le personnage et même par les instrumentistes qui, comme les deux violistes de La Descente d'Orphée aux Enfers de Charpentier, ont pour tâche d'évoquer l'accompagnement de la lyre. C'est un groupe de créateurs qui est en réalité "Orphée " (un Orphée fragmenté, comme démembré par les Bacchantes), tandis l'interprète sur scène doit donner l'illusion d'une unité de création, de l'improvisation spontanée d'un chant magique (un Orphée réunifié et divinisé sous l'égide d'Apollon). Cette division des tâches et cette unité fictionnelle est une belle métaphore de l'opéra. Lorsqu'on fait l'histoire du genre, lorsqu'on fait le récit des péripéties de la conception d'une œuvre lyrique, ou lorsqu'on 
en analyse la partition, on oppose trop souvent la part du librettiste et la part du compositeur, et même celle du premier interprète, on sépare trop souvent les mots et les sons, pour chercher comment la musique "illustre " les paroles. Et devant la partition, on se laisse prendre au piège de la notation occidentale : une ligne pour les notes, une ligne pour les mots, une ou plusieurs lignes pour les instruments. Mais l'expérience du spectacle n'est pas celle-là : dans la performance, le mot est musique, la voix se marie à l'instrument, le chant est incarné, souligné par le geste. Par le biais de l'illusion théâtrale, c'est la magie d'Orphée qui est à l'œuvre et qui unifie un spectacle collectif, fusionnant les arts.

C'est donc un corps, celui de l'interprète, celui de l'Orphée moderne, qui assume le travail collectif. Cette invention baroque du chanteur (et de la chanteuse) d'opéra est fondamentale et oriente une bonne part de la musique qui a suivi, car même l'instrument seul bientôt devra se faire chanteur et mimer le chant pour émouvoir. Et ce n'est pas un hasard si l'évolution de l'opéra baroque tend vers toujours plus de virtuosité vocale. $0 n$ a trop souvent ajouté foi aux propos de Gluck et de Calzabigi dans la fameuse préface d'Alceste, qui critique le dramma per musica baroque pour promouvoir une nouvelle esthétique. Et on a vu l'opéra baroque comme artificiel, excessivement orné et soumis à la tyrannie des chanteurs. L'interprétation de des œuvres baroques, aujourd'hui, et la découverte de leur efficacité théâtrale nous démontre le contraire. La stupeur que doit provoquer le chant d'Orphée n'est pas incompatible avec le théâtre, bien au contraire, ni la virtuosité avec l'émotion. Mais on a trop souvent oublié, dans la quête de réalisme de l'opéra du XIXe et du début du XXe siècle, que le chant lyrique est en lui-même merveilleux, magique, et qu'il ouvre, tel Orphée, des passages entre les mondes.

\section{Notas}

1 Le texte de cette conférence a été conçu pour introduire une représentation de La Descente d'Orphée aux Enfers de Marc-Antoine Charpentier, le 27 octobre 2009, au théâtre de Sabará, MG, dans le cadre de la II Semana de Musica Antiga da UFMG.

Raphaëlle Legrand é professora de história e análise de música barroca na Universidade de Paris-Sorbonne, Paris IV. Publicou, com Nicole Wild, Regards Sur l'opéra-comique, trois siècles de vie théâtrale (CNRS Editions, 2002; premiada pela Académie Charles Cros) e com Laurine Quetin, Entre théâtre et musique: Récitatifs en Europe aux XVIle et XVIIle siècles (Cahiers d'histoire culturelle, Université de Tours, 1999) e a obra Rameau et le pouvoir de l'harmonie (Cité de la Musique, 2007) e numerosos artigos sobre o compositor e téorico. Atualmente escreve uma obra comparativa sobre música e significado nas obras de Bach, Handel, Rameau e Vivaldi. Raphaëlle Legrand é membro de diversos corpos editoriais, como: Société Française de Musicologie, OEuvres Complètes de Lully, e das revistas Musurgia, Analyse et Pratique Musicales e Jardin de Musique. 